\title{
A pilot study on pharmacokinetic/pharmacodynamic target attainment in critically ill patients receiving piperacillin/tazobactam
}

\author{
Jiřina Martínková ${ }^{1}$, Manu L.N.G. Malbrain², Eduard Havel ${ }^{1,3}$, Petr Šafránek', \\ Jan Bezouška3 ${ }^{3}$ Milan Kaška1, 3 \\ ${ }^{1}$ Surgical Department, University Hospital, Hradec Králové, Czech Republic \\ ${ }^{2}$ Intensive Care Unit and High Care Burn Unit, Ziekenhuis Netwerk Antwerpen, ZNA Stuivenberg, Antwerpen, Belgium \\ ${ }^{3}$ Academic Department of Surgery, Medical Faculty, Charles University and Surgical Department, \\ University Hospital, Hradec Králové, Czech Republic
}

\begin{abstract}
Background: In critically ill patients, multi-trauma and intensive therapy can influence the pharmacokinetics (PK) and pharmacodynamics (PD) of antibiotics with time-dependent bacterial killing. Consequently, PK/PD targets $\left(\% f \mathrm{f}_{>\mathrm{MIC}}\right)$ — crucial for antimicrobial effects -may not be attained.

Methods: Two patients admitted to the surgical ICU of the University Hospital in Hradec Králove for multiple-trauma were given piperacillin/tazobactam by 1-hour IV infusion 4/0.5 g every $8 \mathrm{~h}$. PK variables: total and renal clearance $\left(\mathrm{CL}_{\text {tot }}\right.$ $\left.\mathrm{CL}_{\mathrm{R}}\right)$, volume of distribution $\left(\mathrm{V}_{d}\right)$, and elimination half-life $\left(\mathrm{T}_{1 / 2}\right)$ were calculated, followed by glomerular filtration rate (MDRD) and cumulative fluid balance (CFB-total fluid volume based on 24-h registered fluid intake minus output). The PK/PD target attainment $\left(100 \% f \mathrm{~T}_{>M I C}\right)$ was defined as free $(f)$ piperacillin plasma concentrations that remain, during the entire dosing interval $(\mathrm{T})$, above the minimum inhibitory concentration $\left(100 \% \mathrm{fT}>_{\text {MIC }}\right.$ ) within days $4-8$ (when CFB culminates and disappears). Piperacillin concentrations were determined by liquid chromatography-tandem mass spectrometry (LC-MS/MS) and corrected for unbound fraction (22\%).

Results: CFB culminated over days $2-5$ reaching $15-30 \mathrm{~L}$ and was associated with a large $\mathrm{V}_{\mathrm{d}}(29-42 \mathrm{~L})$. While MDRD in patient 1 was low $\left(0.3-0.4 \mathrm{~mL} \mathrm{~s}^{-1} 1.7 \mathrm{~m}^{-2}\right)$, that of patient 2 was increasing $\left(>3.1 \mathrm{~mL} \mathrm{~s}^{-1} 1.7 \mathrm{~m}^{-2}\right)$, which was associated with augmented $\mathrm{CL}_{\mathrm{R}}$. In patient 2 , the $f T$ reached only 62,52 , and $44 \%$ on days 4,6 , and 8 , respectively. In patient 1 , the $\% f T$ was much higher, attaining values four to fivefold greater than that targeted.

Conclusions: Critically ill patients are at risk of drug under- or overdosing without dose up-titration with regard to covariate effects and individual drug pharmacokinetics.
\end{abstract}

Key words: PK/PD target attainment, body fluid retention, pharmacokinetics, pharmacodynamics, piperacillin, critically ill patients, personalised therapy

Anaesthesiology Intensive Therapy 2016, vol. 48, no 1, 23-28

Antibiotic dosing regimens are usually based on healthy volunteers with normal physiology or non-critically ill patients. In critically ill hospitalized patients, pathophysiological changes may have profound effects on the primary determinants of the pharmacokinetics (PK) of hydrophilic antibiotics which are distributed to interstitial fluid and are predominantly excreted via the kidneys ( $\beta$-lactams, amino- glycosides, glycopeptides). Extravascular volume expansion with fluid loading and capillary leak may alter their low volume of distribution $\left(V_{d}\right)$, while changes in renal function can significantly influence drug clearance (Cl) [1].

The $\beta$-lactams are the most commonly prescribed antibiotics in the critically ill. Since bacterial killing is considered time-dependent, the PD parameter of interest is the 
fraction of time that the free drug's concentration exceeds a minimum inhibitory concentration (MIC tested in vitro) of the causative microorganism - the $f \mathrm{~T}_{>\mathrm{MIC}}$.

It is traditionally accepted that maintaining concentrations above the MIC of the causative organisms around $40-70 \%$ of the time (during each dosing interval) is adequate $\left(40-70 \% f \mathrm{~T}_{>\mathrm{MIC}}\right)$ [2]. However, recent studies suggest that higher targets are needed in critically ill patients to improve clinical outcome whilst minimizing the development of resistance. Concentrations four to fivefold greater than the MIC are associated with maximum bactericidal activity $[3,4]$. The PK/PD target was set at $100 \% f \mathrm{~T}>4-5 \times \mathrm{MIC}$.

In this prospective, observational, pharmacokinetic report, the PK/PD target was set at $100 \% \mathrm{fT}_{>\mathrm{MIC}}$ (the piperacillin breakpoint for Pseudomonas aeruginosa being $16 \mu \mathrm{g}$ $\mathrm{mL}^{-1}$ - according to EUCAST). The primary endpoint of this pilot study is the documentation of differences within and between subjects in reaching the target PK/PD attainment in two intensive care unit (ICU) trauma patients who developed a positive cumulative fluid balance (CFB) during treatment with a standard dose of piperacillin. Intensive crystalloid fluid loading in ICU patients according to Rivers "early goal-directed therapy" [5] is one possible cause of a positive CFB during the first posttraumatic days. Moreover, the PK/PD target needs to be analysed throughout the ICU stay because of the dynamic nature of critical illness. In the past, a positive CFB and glomerular filtration rate were the main pathophysiological variables monitored in relation to PK/PD. Secondary endpoints were maximum absolute values of $f \mathrm{~T}_{>M \mathrm{MIC}}$ attained.

\section{METHODS}

Patient 1 (male) and patient 2 (female), were admitted in the ICU of the Surgical Clinic, at the Teaching Hospital, Hradec Králové, the Czech Republic, for multiple-trauma. The demographic characteristics for patient 1 were as follows: age 32 years, weight $70 \mathrm{~kg}$, serum creatinine $158 \mu \mathrm{mol} \mathrm{L}{ }^{-1}$, with MDRD $0.74 \mathrm{~mL} \mathrm{~s}^{-1} 1.73 \mathrm{~m}^{-2}$, ISS 54, and affected, apart from skeletal injuries, with left kidney disruption needing acute nephrectomy. Those for patient 2 were as follows: age 59 years, weight $60 \mathrm{~kg}$, serum creatinine $57 \mu \mathrm{mol} \mathrm{L}^{-1}$, with MDRD $1.57 \mathrm{~mL} \mathrm{~s}^{-1}$ $1.73 \mathrm{~m}^{-2}$, ISS 54. They were both treated with piperacillin/ tazobactam as a 1-hour IV infusion of 4/0.5 g given every 8 hours according to the standard protocol for empiric antibiotic treatment of suspected hospital-associated infections and/or ventilator-associated pneumonia during the first week of stay in a surgical ICU, University Hospital in Hradec Kralove (the Czech Republic). All patients were given IV volume-therapy per protocol in order to prevent hypotension [5].

\section{SAMPLING AND $\beta$-LACTAM ASSAY}

Four serial plasma concentrations were obtained on days 6 and 8 (patient 1) and 4, 6, and 8 (patient 2). Blood samples were taken at the baseline ( $0.5 \mathrm{~h}$ prior to the dose) and $1,2.5$, and 4.5 hours post-administration. In patient 1 , blood samples were taken $1.5,3$, and 5.5 hours post-administration on day 6 . Specimens were centrifuged at $3000 \mathrm{rpm}$ for 10 minutes within 30 minutes of sampling and frozen at $-80^{\circ} \mathrm{C}$. Determination of piperacillin in plasma samples was performed using the liquid chromatography-tandem mass spectrometry (LC-MS/MS) modified method [6]. Unbound concentrations were calculated using published studies on the fraction of protein binding for the study drug [7]. Urine samples were taken from a 1-8 hour and 24-hour urine collection in order to calculate cumulative renal excretion of piperacillin and creatinine clearance $\left(\mathrm{Cl}_{\mathrm{Cr}}\right)$, respectively.

\section{PHARMACOKINETIC ANALYSIS}

Each plasma concentration-time plot was based on four points of piperacillin plasma concentrations obtained within the 1-8 hour dosing intervals. Three concentrations were detected using LC-MS/M, the fourth concentration $\left(C_{\min }\right)$ was calculated using the following equation: $C_{t}=C_{0} \times e^{-k t}$ [8]. $C_{0}$ was the $y$-intercept of a plot of piperacillin plasma concentration versus time on the semilog scale. Area under the curve $\left(A \cup C_{1-8}\right.$ ) was estimated using the trapezoidal rule. Elimination half-life was calculated as follows: $\left(T_{1 / 2}\right)=\ln 2 / k_{e^{\prime}}$ where $k_{e}$ is elimination rate constant. The $k_{e}$ was calculated as the negative slope of the non-weighted squares curve fit of three sampling points during post-administration. The apparent volume of distribution $\left(V_{d}\right)$ was calculated from the equations: $\mathrm{V}_{\mathrm{d}}=\mathrm{Cl} / \mathrm{k}_{\mathrm{e}}$. The total clearance $\left(\mathrm{Cl}_{\text {tot,pip }}\right)$ values were estimated as follows: $\mathrm{Cl}_{\text {tot }}=$ dose/AUC $_{1-8^{\prime}}$ and renal clearance $\left(\mathrm{Cl}_{\mathrm{R} \text {,pip }}\right)=\mathrm{TADU}_{1-8} / \mathrm{AUC}_{1-8}$. TADU ${ }_{1-8}$ is the amount of piperacillin excreted in the urine collected over 1-8 hour post-administration, while $\mathrm{AUC}_{1-8}$ is the area under the plasma concentration-time curve within the timespan $t_{1}$ (after termination of the 1-hour infusion) to $t_{8}$ (at the end of a dosing interval). Renal function was defined in the Central Laboratory University Hospital, Hradec Králové as a glomerular filtration rate (eGFR) assessed by a value of MDRD [9] and creatinine clearance $\left(\mathrm{Cl}_{\mathrm{Cr}}\right)$.

\section{PHARMACODYNAMIC ANALYSIS}

The $f \mathrm{~T}_{>M \mathrm{MI}}$ was calculated for both the total and unbound (free) piperacillin by observing the time during the dosing interval that the log-linear least squares regression profile of drug concentrations intersected the target MIC for Pseudomonas aeruginosa, based on EUCAST breakpoints ( $16 \mu \mathrm{g}$ $\mathrm{mL}^{-1}$ for piperacillin) [10]. The target was $100 \% \mathrm{fT}_{>M I C^{\prime}}$ i.e. free piperacillin concentration maintained above the breakpoint value throughout the entire dosing interval. CFB was evalu- 

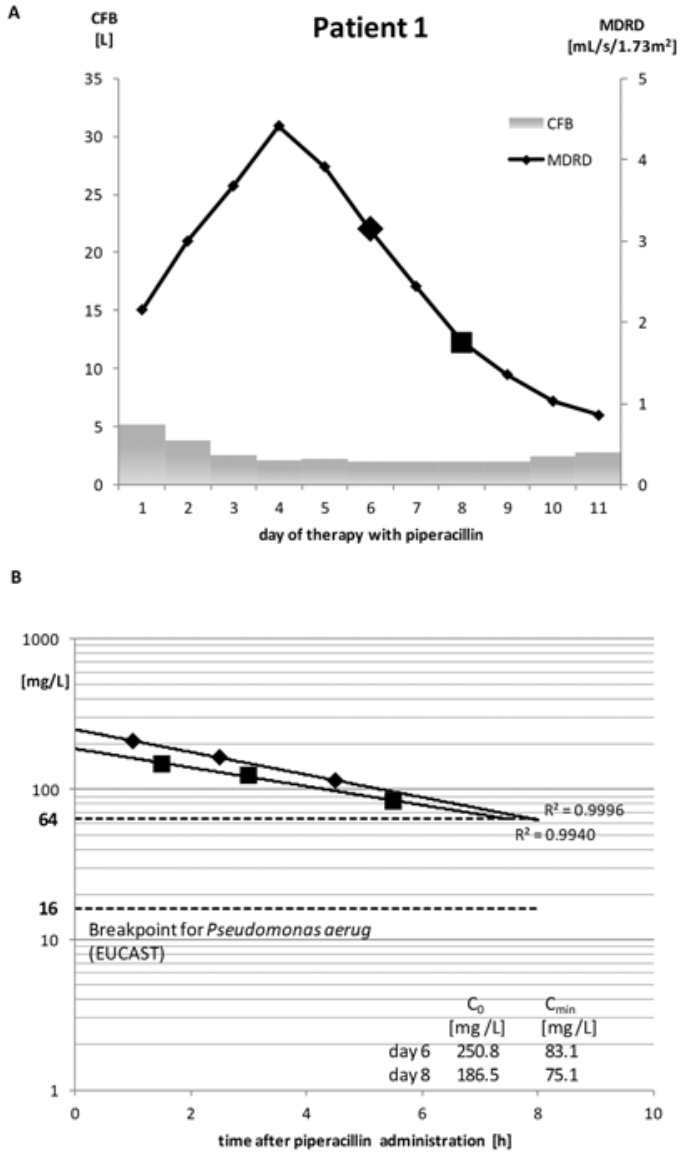

Figure 1. Patient 1

Panel A. Cumulative fluid balance (CFB) and glomerular filtration rate (MDRD). Panel B. Total piperacillin plasma concentration $\bullet$ time plots on day 6 , and 8 of therapy

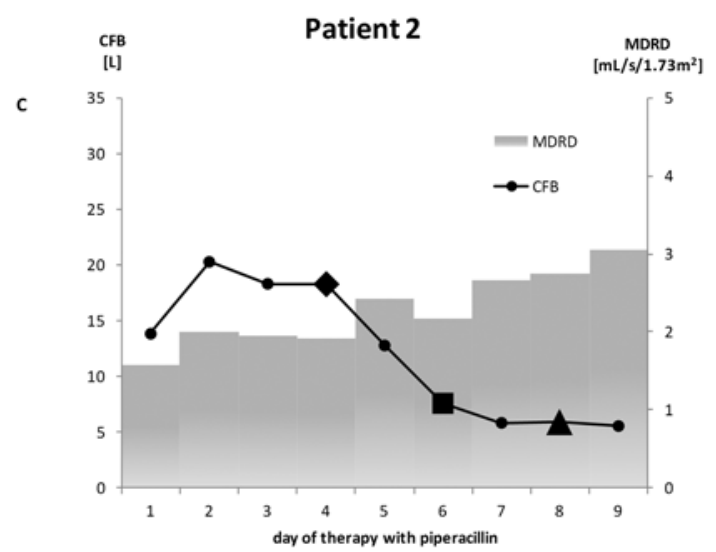

D

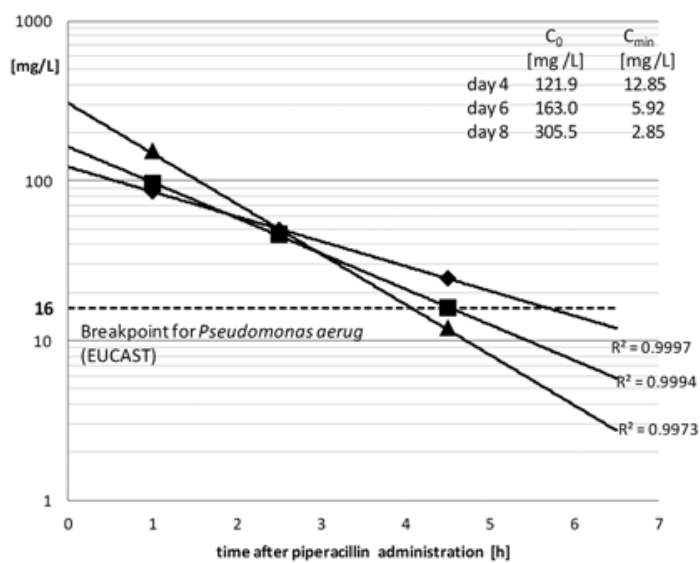

Figure 2. Patient 2

PanelC. Cumulative fluid balance (CFB) and glomerular filtration rate (MDRD). PanelD. Total piperacillin plasma concentration $\bullet$ time plots on day 4,6 , and 8 of therapy.

Figure 1. Cumulative fluid balance and glomerular filtration rate (MDRD) in relation to the target PK/PD attainment in patient 1 (panel A, B) and patient 2 (panel C, D). The volume of CFB culminated over days 2-5 (patient 1 and 2) of antibiotic treatment. While glomerular filtration rate (GFR) in patient 2 is high (as a sign of augmented renal clearance syndrome), that in patient 1 is very low as a consequence of severe renal injury. Attainment of the $f T$ is based on a plasma-concentration-time plot in the semi-log graph and its intersection with the line of $16 \mathrm{mg} \mathrm{L}^{-1}$ (the piperacillin breakpoint for Pseudomonas aeruginosa based on EUCAST). Piperacillin plasma concentrations were measured on days 6 and 8 (patient 1 ) and days 4,6 , and 8 (patient 2). Patient 2 failed to attain the maximum PK/PD target $\left(100 \% \mathrm{fT}>\mathrm{MIC}\left(16 \mathrm{mg} \mathrm{mL}^{-1}\right)\right.$. Moreover, the $f \mathrm{~T}$ decreases with the number of days of treatment. On the $x$-axis of the semi-log graph, this value is gradually shifted to the left. In contrast, in patient 1 an unexpected

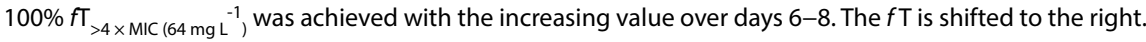

ated daily as the difference between fluid intake and output. Free piperacillin concentration maintaining a level above16 $\mu \mathrm{g} \mathrm{mL}^{-1}$ during a dosing interval was considered the target attainment. The glomerular filtration rate was assessed by the MDRD equation [9], while every $\mathrm{Cl}_{\mathrm{cr}}$ was examined on days when PK parameters were estimated, as follows:

24-hour $\mathrm{Cl}_{\mathrm{cr}}=\mathrm{U}_{\mathrm{v}} \times \mathrm{U}_{\mathrm{cr}} /\left(1.440 \times \mathrm{S}_{\mathrm{cr}}\right)$, where $\mathrm{U}_{\mathrm{v}}$ is urinary volume $(\mathrm{L}), \mathrm{U}_{\mathrm{cr}}$ the urinary creatinine concentration $\left(\mu \mathrm{mol} \mathrm{L} \mathrm{L}^{-1}\right)$, and $\mathrm{S}_{\mathrm{cr}}$ the serum creatinine concentration $\left(\mu \mathrm{mol} \mathrm{L} \mathrm{L}^{-1}\right)[11]$.

\section{RESULTS}

The sampling method used allowed one to describe a mono-exponential, first-order elimination process [5].
Patient 1 had a low GFR as demonstrated with MDRD (Fig. 1, Panel A) and a very positive CFB. The estimated $\mathrm{Cl}_{\mathrm{cr}}$ was 9 and $15 \mathrm{~mL} \mathrm{~min}^{-1}$ on days 6 and 8, respectively. On those days, a $100 \% \mathrm{fT}_{>\mathrm{MIC}}$, as well as a $100 \% \mathrm{fT}_{>4 \times \mathrm{MIC}\left(64 \mathrm{mg} \mathrm{L}^{-1}\right)}$ was reached (Fig.1, Panel B). The slope of total piperacillin plasma concentration indicates a very low $\mathrm{k}_{\mathrm{e}}$ value (Table 1 ).

In contrast, the results obtained from patient $2 \mathrm{dem}$ onstrated increasing GFR presented as daily MDRD, and positive values of CFB (Fig. 1, Panel C). The estimated $\mathrm{Cl}_{\mathrm{cr}}$ was 112,124 , and $145 \mathrm{~mL} \mathrm{~min}^{-1}$ on days 4,6 , and 8 , respectively.

Patient 2 failed to attain the maximum PK/PD target $\left(100 \% f T_{>M I C}\right.$ ) (Fig. 1, Panel D). The $f T$ attained 80, 65, and $55 \%$ if derived from total piperacillin plasma concentrationtime plots on days 4, 6, and 8, respectively (Fig. 1, Panel D). 
Table 1. Piperacillin pharmacokinetics in intensive care unit patients Nos.1 and 2

\begin{tabular}{lccccc}
\hline PK parameters of unbound drug* & $\mathrm{V}_{\mathrm{d}}[\mathrm{L}]$ & $\mathrm{k}_{\mathrm{e}}\left[\mathrm{h}^{-1}\right]$ & Clearance $_{\text {tot, pip }}\left[\mathrm{L} \mathrm{h}^{-1}\right]$ & Clearance $_{\mathrm{R}, \mathrm{pip}}\left[\mathrm{L} \mathrm{h}^{-1}\right]$ & $\mathrm{t}_{1 / 2}[\mathrm{~h}]$ \\
\hline Patient 1 & & & & & \\
Day 6 & 27.5 & 0.157 & 7.17 & 4.6 & 4.4 \\
Day 8 & 21.1 & 0.178 & 6.50 & & 3.9 \\
Patient 2 & & & & 7.1 & 2.1 \\
Day 4 & 42.1 & 0.333 & 16.2 & 11.5 & 1.4 \\
Day 6 & 27.6 & 0.498 & 15.4 & 10.2 & 0.9 \\
Day 8 & 11.3 & 0.736 & 11.3 & & \\
\hline
\end{tabular}

PK - pharmacokinetic; Clearance $_{\text {tot,pip }}$ - total plasma clearance; Clearance $_{\mathrm{R}, \text { pip }}$ - renal clearance; ${ }^{*}$ observed plasma concentrations of piperacillin were corrected for binding with plasma protein (22\%); $\mathrm{Vd}$ - volume of distribution; $\mathrm{k}_{\mathrm{e}}$ - elimination rate constant; $\mathrm{t}_{1 / 2}$ - elimination half time

Total piperacillin plasma concentration plots (days 4, 6, and 8) differ with regard to the slope, as demonstrated by the $k_{e}$ value (Table 1).

The $f T$ estimated for unbound piperacillin plasma concentrations in patient 1 maintained a value of $100 \% f \mathrm{fT}_{>4 \times \mathrm{MIC}}$ (64 mg mL${ }^{-1}$ ), while in patient 2 the $f \mathrm{~T}$ achieved 62,52 , and $44 \% \mathrm{fT}_{>\mathrm{MIC}}$ on days 4,6 , and 8 , respectively. In this patient, the pharmacokinetic variables of piperacillin show increasing values of $\mathrm{Cl}_{\mathrm{R} \text {,pip }}$ in accordance with $\mathrm{Cl}_{\mathrm{cr}^{\prime}}$, while decreasing values were observed for $\mathrm{Cl}_{\text {tot,pip }}, \mathrm{V}_{\mathrm{d}^{\prime}}$, and $\mathrm{t}_{1 / 2}$ over days 4-8 of treatment (Table 1).

\section{DISCUSSION}

This pilot study reports the results on the first two typical patients studied in our institution. In order to describe intraindividual variability in the target PK/PD attainment, we have studied these 2 patients whose clinical status mainly differed with regard to renal function, while CFB was similar.

The dynamic nature of critical illness does not allow one to achieve a predictable "steady state". $\mathrm{Cl}_{\text {tot }}$ and apparent $\mathrm{V}_{\mathrm{d}}$ are considered two PK parameters that closely correlate with adequate drug dosing. $V_{d}$ determines the initial drug dose, whereas the maintenance dose (in order to maintain a "steady state" - plasma concentration) is based on clearance.

$V_{d}$ is the factor that relates the amount of a drug in the body to the concentration of the drug in the sampling site. This hypothetical volume is determined by the drug's distribution behaviour. For hydrophilic drugs, this value is thought to be variable in critically ill patients with acute kidney injury and sepsis. Sepsis can lead to the development of endothelial damage followed by increased capillary permeability, which results in the displacement of intravascular fluid from the systemic circulation into the interstitium [12]. These fluid shifts into the interstitial space can be substantial owing to the large fluid volumes often administered to septic patients as a part of resuscitation (fluid overload) [13].
Extravascular volume can be even more marked in patients who have a decreased urine output secondary to acute kidney injury [14-16]. This fluid related weight gain correlates closely with increased mortality [14]. Moreover, an increase in fluid volume can dilute serum creatinine concentration, resulting in inappropriately low measures, and delay diagnosis of acute kidney insufficiency (AKI) [17].

These changes in PK variables were first described for hydrophilic aminoglycosides whose bactericidal activity is concentration dependent. Maximum concentration $\left(C_{\max }\right)$ of gentamicin detected 1 hour after a 30-minute IV infusion should be 8 to 12 times higher than the MIC tested in vitro $[18,19]$. In ICU patients with sepsis the $V_{d}$ of gentamicin was $0.48 \mathrm{~L} \mathrm{~kg}^{-1}$, compared to $0.29 \mathrm{~L} \mathrm{~kg}^{-1}$ in postoperative non-septic patients [20]. With respect to dosing, an increase in $\mathrm{V}_{\mathrm{d}}$ should be taken into account with an adapted (increased) loading dose to achieve a concentration within the therapeutic range and to avoid underdosing. On the other hand, a maintenance dose dependent on the clearance of a drug is necessary to maintain a "steady state". As the patient's clinical status improves and the degree of tissue oedema disappears, the value of $V_{d}$ can decrease dramatically within days. The drug dosage should be adjusted again in order to prevent drug accumulation and overdosing. This was shown during treatment with gentamicin in critically ill patients with suspected or confirmed sepsis related to a Gram-negative bacterial infection. Over days 2-7 of treatment (after aggressive fluid loading), the $V_{d}$ of gentamicin decreased from $0.43 \mathrm{~L} \mathrm{~kg}^{-1}$ to $0.29 \mathrm{~L} \mathrm{~kg}^{-1}$. The required dosage was reduced from $5.14 \mathrm{mg} \mathrm{kg}^{-1}$ every $24 \mathrm{~h}$ to $3.98 \mathrm{mg}$ every $24 \mathrm{~h}$ despite the patient's renal functions remaining stable [21].

Comparable changes in PK variables in critically ill ICU patients have also been described in clinical studies using hydrophilic time-dependent piperacillin (a beta-lactam), and meropenem (carbapenem). Time-dependency is based on the PK/PD target attainment, i.e. on the time (percentage of the dosing interval) covered by antibiotic plasma 
concentration exceeding the MIC tested in vitro. Recently, it was found that many ICU patients experienced an augmented renal clearance syndrome (ARC) characterized by

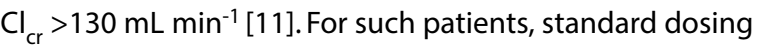
of antibiotics eliminated predominantly "via the kidneys", was not adequate [10, 22-24]. In spite of the fact, that the mechanisms for ARC have not been fully clarified yet, it is considered an independent predictor for not achieving the PK/PD target [6]. This implies that more aggressive dosing regimens should be used in such patients. On the other hand, low $\mathrm{Cl}_{\mathrm{cr}}$ may be predictive for insufficient renal excretion of piperacillin and risk of drug toxicity.

Within this respect, future techniques (like bio-electrical impedance analysis, BIA) to assess fluid overload and volume excess may be of special interest in critically ill patients [25].

Our findings are in good agreement with the clinical outcomes mentioned above. Patient 2 did not reach the predefined PK target $\left(100 \% \mathrm{fT}_{>\mathrm{MIC}}\right)$ probably related to his high $\mathrm{Cl}_{\mathrm{cr}}\left(\geq 112 \mathrm{~mL} \mathrm{~min}^{-1}\right)$. In addition, over time the $\mathrm{fT}$ was decreasing. For this patient, standard dosing was not adequate and resulted in subtherapeutic plasma concentrations (drug underdosing). In contrast, patient 1 had a low $\mathrm{Cl}_{\mathrm{cr}}$ and attained unexpected values of $100 \% \mathrm{fT}_{>4 \times \mathrm{MIC}}$. At a standard dosing regimen, the target $100 \% \mathrm{fT}>4 \times \mathrm{MIC}$ can only be attained in case of a low $\mathrm{Cl}_{\mathrm{cr}}$ and consequently a low $\mathrm{Cl}_{\mathrm{R}, \mathrm{pip}}$. Hence, this patient was at risk of drug toxicity [26]. Although considered safe, beta-lactam antibiotics have a number of adverse effects including neurotoxicity, liver damage and bone marrow suppression [26]. Moreover, it should be stressed that piperacillin dosing for this patient should have been reduced at the beginning of treatment and individually adjusted afterwards [27]. We have noticed that with repeated standard piperacillin doses given in multiple trauma patients having a low $\mathrm{Cl}_{\mathrm{cr}}$ due to acute kidney injury, the $f \mathrm{~T}_{>\mathrm{MIC}}$ expressed on $\mathrm{x}$-axis on the semi-log graph will be shifted to the right while in a multiple trauma patient with ARC, the $f T_{>M I C}$ will be shifted to the left. This is important in clinical practice, as intra-individual changes in PK variables during treatment imply that dose adjustment may be warranted.

In addition, critically ill patients with fluid overload and AKI often experience larger $V_{d}$ for hydrophilic antibiotics related to decreased binding of the drug to serum proteins (not analysed in this report). Although this can be explained not only by the reduced albumin synthesis, it could also be a result from the accumulation of certain uremic molecules that may compete with other drugs for the same albumin binding sites. An increase in $V_{d}$ in patients with AKI has been also documented for hydrophilic amikacin, meropenem, and vancomycin [27].

Changes in PK parameters are related to changes in the PK/PD target. $\mathrm{Cl}_{\mathrm{R} \text {, pip }}$ increases in accordance with $\mathrm{Cl}_{\mathrm{Cr}}$ [5], while $\mathrm{Cl}_{\text {tot,pip }}$ decreases when there is a rapid fall in $\mathrm{V}_{d}$ and a slow decrease in $\mathrm{T}_{1 / 2}$, as indicated by $\mathrm{Cl}_{\text {tot }}=\ln 2 \times \mathrm{V}_{\mathrm{d}} / \mathrm{T}_{1 / 2}$.

\section{CONCLUSION}

Without individual dose adjustment, critically ill patients are at risk of either treatment failure (underdosing), or drug toxicity (overdosing). Thus, a dosing regimen should be based on repeated therapeutic drug monitoring and personalised antibiotic treatment.

\section{ACKNOWLEDGMENTS}

1. This research was supported by the Internal Grant Agency (IGA) of the Ministry of Health, Czech Rep.: IGA MZ - NT14089-3/2013.

2. The authors declare no conflict of interest.

\section{References:}

1. Roberts DM, Roberts JA, Roberts MS et al.; Renal Replacement Therapy Study Investigators: Variability of antibiotic concentrations in critically ill patients receiving continuous renal replacement therapy: a multicentre pharmacokinetic study. Crit Care Med 2012; 40: 1523-1528. doi: 10.1097/CCM.0b013e318241e553.

2. De Waele J J. Carrette S, Carlier M et al.: Therapeutic drug monitoring-based dose optimisation of piperacillin and meropenem: a randomised controlled trial. Intensive Care Med 2014; 40: 380-387. doi: 10.1007/s00134-013-3187-2.

3. Hites $M$, Taccone FS, WolffF et al.: Case-control study of drug monitoring of $\beta$-lactams in obese critically ill patients. Antimicrob Agents Chemother 2013; 57: 708-715. doi: 10.1128/AAC.01083-12.

4. Bauer SR, Salem C, Connor MJ Jr et al.: Pharmacokinetics and pharmacodynamics of piperacillin-tazobactam in 42 patients treated with concomitant CRRT. Clin J Am Soc Nephrol 2012; 7: 452-457. doi: 10.2215/CJN.10741011.

5. Rivers E, Nguyen B, Havstad S et al.; Early Goal-Directed Therapy Collaborative Group: Early goal-directed therapy in the treatment of severe sepsis and septic shock. N Engl J Med 2001; 345: 1368-1377.

6. Ohmori T, Suzuki A, Niwa T et al.: Simultaneous determination of eight $\beta$-lactam antibiotics in human serum by liquid chromatography-tandem mass spectrometry. J Chromatogr B Analyt Technol Biomed Life Sci 2011; 879: 1038-1042. doi: 10.1016/j.jchromb.2011.03.001.

7. Tjandramaga TB, Mullie A, Verbesselt $R$, De Schepper PJ, Verbist L: Piperacillin: human pharmacokinetics after intravenous and intramuscular administration. Antimicrob Agents Chemother 1978; 14: 829$-837$.

8. Hedaya MA: Basic pharmacokinetics. Pharmacy education series. CRC Press. Taylor\& Francis Group, Boca Raton-London-NewYork 2007.

9. Gaber AO, Moore LW, Aloia TA et al.: Cross-sectional and case-control analyses of the association of kidney function staging with adverse postoperative outcomes in general and vascular surgery. Ann Surg 2013; 258: 169-177. doi: 10.1097/SLA.0b013e318288e18e.

10. Levey $A S$, Inker $L A$, Coresh J: GFR estimation: from physiology to public health. Am J Kidney Dis 2014; 63: 820-834. doi: 10.1053/j. ajkd.2013.12.006.

11. Carlier M, Carrette $S$, Roberts JA et al.: Meropenem and piperacillin/tazobactam prescribing in critically ill patients: does augmented renal clearance affect pharmacokinetic /pharmacodynamic target attainment when extended infusions are used? Crit Care 2013; 17: R84. doi: 10.1186/cc12705.

12. Boucher BA, Wood GC, Swanson JM: Pharmacokinetic changes in critica illness. Crit Care Clin 2006; 22: 255-271.

13. Malbrain ML, Marik PE, Witters I et al: Fluid overload, de-resuscitation and outcomes in critically ill or injured patients: a systematic review with suggestions for clinical practice. Anaesthesiol Intensive Ther 2014; 46: 361-380. doi: 10.5603/AIT.2014.0060.

14. Fülöp T, Pathak MB, Schmidt DW et al.: Volume-related weight gain and subsequent mortality in acute renal failure patients treated with continuous renal replacement therapy. ASAIO J 2010; 56: 333-337. doi: 10.1097/MAT.0b013e3181de35e4. 
15. Schmidt C, Höcherl K, Schweda F, Bucher M: Proinflammatory cytokines cause down-regulation of renal chloride entry pathways during sepsis. Crit Care Med 2007; 35: 2110-2119.

16. Morrell ED, Kellum JA, Hallows KR, Pastor-Soler NM: Epithelial transport during septic acute kidney injury. Nephrol Dial Transplant 2014; 29: 1312-1319. doi: 10.1093/ndt/gft503.

17. Macedo E, Bouchard J, Soroko SH et al.: Fluid accumulation, recognition and staging of acute kidney injury in critically-ill patients. Program to Improve Care in Acute Renal Disease Study. Crit Care 2010; 14: R82. doi:10.1186/cc9004.

18. Moore RD, Smith CR, Lietman PS: The association of aminoglycoside plasma levels with mortality in patients with gram-negative bacteremia. J Infect Dis 1984; 149: 443-448.

19. Moore RD, Smith CR, Lietman PS: Risk factors for the development of auditory toxicity in patients receiving aminoglycosides. J Infect Dis 1984; 149: 23-30.

20. Tang GJ, Tang JJ, Lin BS, Kong CW, Lee TY: Factors affecting gentamicin pharmacokinetics in septic patients. Acta Anaesthesiol Scand 1999;43:726-730.

21. Triginer C, Izquierdo I, Fernández R et al.: Gentamicin volume of distribution in critically ill septic patients. Intensive Care Med 1990; 16: 303-306.

22. Udy AA, De Waele JJ, Lipman J: Augmented renal clearance and therapeutic monitoring of $\beta$-lactams. Int J Antimicrob Agents 2015; 45: 331-333. doi: 10.1016/j.jijantimicag.2014.12.020.

23. Udy $A A$, Jarrett $P$, Stuart J et al.: Determining the mechanisms underlying augmented renal drug clearance in the critically ill: use of exogenous marker compounds. Crit Care 2014;18: 657-656. doi: 10.1186/s13054014-0657-z.
24. Udy AA, Lipman J, Jarrett $P$ et al.: Are standard doses of piperacillin sufficient for critically ill patients with augmented creatinine clearance? Crit Care 2015; 19: 28. doi: 10.1186/s13054-015-0750-y.

25. Malbrain ML, Huygh J, Dabrowski W, De Waele JJ, Staelens A, Wauters J: The use of bio-electrical impedance analysis (BIA) to guide fluid management, resuscitation and deresuscitation in critically ill patients: a bench-to-bedside review. Anaesthesiol Intensive Ther 2014; 46: 81-91, doi: 10.5603/AIT.2014.0061.

26. De Waele JJ, Lipman J, Akova $M$ et al.: Risk factors for target non-attainment during empirical treatment with $\beta$-lactam antibiotics in critically ill patients. Intensive Care Med 2014; 9: 1340-1351. doi: 10.1007/s00134-014-3403-8.

27. Eyler RF, Mueller BA: Antibiotic dosing in critically ill patients with acute kidney injury. Nat Rev Nephrol 2011; 7: 226-235. doi: 10.1038/nrneph.2011.12

\section{Corresponding author:}

Milan Kaška, Assoc Prof, PhD, MD,

Surgical Department, University Hospital,

Sokolská 581, 50005 Hradec Králové, Czech Republic

e-mail:kaskam@lfhk.cuni.cz

Received: 24.08.2015

Accepted: 18.11.2015 